\title{
An investigation of cortical neuroplasticity following stroke in adults: is there evidence for a critical window for rehabilitation?
}

Michelle N. McDonnell ${ }^{1 *}$, Simon Koblar², Nick S. Ward ${ }^{3,4,5}$, John C. Rothwell ${ }^{3}$, Brenton Hordacre ${ }^{6}$ and Michael C. Ridding ${ }^{6}$

\begin{abstract}
Background: Evidence in animal stroke models suggests that neuroplasticity takes place maximally in a specific time window after an ischaemic lesion, which may coincide with the optimal time to intervene with rehabilitation. The aim of this study is to investigate neurophysiological evidence for a "critical window" of enhanced neuroplasticity in patients following ischaemic stroke, and establish its duration. We will also investigate changes in cortical inhibition following stroke, and the influence this has on functional recovery.

Methods/Design: We will recruit participants recently admitted to the Stroke Unit of major metropolitan hospitals who have had a stroke and can provide informed consent. Participants will be excluded if they have any contraindications to Transcranial Magnetic Stimulation. We will compare neurophysiological outcomes in an age-matched healthy control group. We conservatively hypothesise a $5 \%$ increase in neuroplasticity at the optimal timing following stroke, compared to control participants, and require 43 patients following stroke to detect a significant difference with $80 \%$ power. The primary outcome is the change in the motor evoked potential (MEP) amplitude in a hand muscle, after the administration of a plasticity-inducing paradigm to the affected hemisphere. Secondary outcomes include measures of cortical excitability, intracortical inhibition and arm function.

Discussion: The data from this trial will clarify whether there is a critical window for neuroplastic change in the brain following stroke. If so, intensive rehabilitation during this period could be more effective, reducing long-term disability and the cost burden of stroke.
\end{abstract}

Keywords: Stroke, Neuroplasticity, Critical window

\section{Background}

Following stroke, a number of structural changes take place in the cortex that facilitate true recovery (restitution) of function. For example, there is evidence for a short period of new axonal growth in areas surrounding a cortical ischaemic lesion [1]. This allows for some active replacement of connectivity that has been damaged by the stroke. Maximising this neuroplasticity is likely to be an important avenue for optimising functional recovery.

There is overwhelming evidence that neuroplastic mechanisms are available throughout life and play an important

\footnotetext{
* Correspondence: michelle.mcdonnell@unisa.edu.au

'Alliance for Research in Exercise, Nutrition and Activity and International Centre for Allied Health Evidence, Sansom Institute for Health Research, School of Health Sciences, University of South Australia, GPO Box 2471, Adelaide, SA 5001, Australia

Full list of author information is available at the end of the article
}

part in stroke recovery [2]. However, there are also 'critical periods' during development in which neuroplastic capabilities are greatest and environmental/behavioural experience during these times results in major changes in structure and connectivity within the brain [3].

Direct evidence of such 'critical period'-like neuroplasticity following stroke is limited in humans. However, neuroimaging and non-invasive stimulation techniques have provided important clues that such an enhanced period of neuroplasticity might be present [4-6]. It might be expected that rehabilitative interventions applied during this period would yield the best functional outcomes.

There are additional changes in the post-stroke brain that might affect neuroplasticity and influence recovery. Hyperexcitability occurs in the area surrounding a cortical lesion 


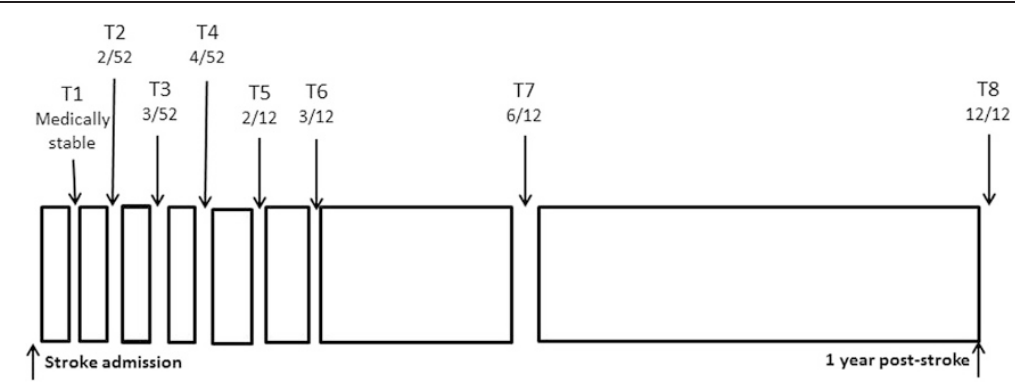

Fig. 1 Timeline of assessments

and is associated with greater long-term potentiation and reduced gamma-aminobutyric acid (GABA) mediated inhibition [7, 8]. Human data also suggests that GABAergic inhibition is reduced in the stroke affected hemisphere $[9,10]$. Modulations in inhibition might influence functional neuroplasticity in the post-stroke brain.

Some behavioural data in humans suggest that there might be optimal windows for rehabilitation post stroke; the earlier that patients are admitted to stroke rehabilitation, the greater the functional gains [11]. In order to optimise the timing of post-stroke therapy, it is critical to determine the time-course of neuroplastic change within the stroke-damaged cortex.

\section{Methods/Design}

\section{Objective}

The primary aim of this study is to provide neurophysiological evidence of a critical window of enhanced neuroplasticity following stroke, and to determine whether the rate of functional recovery is greatest during the period of enhanced neuroplasticity. We anticipate that recovery may be linked to changes in cortical inhibition following stroke, and we will investigate this as a secondary aim of the study.

\section{Design}

This study is a prospective cohort study of adults following a first-ever stroke. We will recruit participants from metropolitan hospitals in Adelaide, Australia and London, England.

\section{Patient population}

We will recruit adults over the age of 18 with no upper age limit into the study. The detailed study inclusion and exclusion criteria are outlined in Table 1.

We will also recruit 35 age- and sex-matched healthy control participants to take part in the neurophysiological measures described below on three occasions, separated by six months. They will be included if they have no history of neurological disorders, and excluded if they have any contraindications to TMS. All participants will provide written, informed consent in accordance with the Declaration of
Helsinki. The study procedures have been approved by the relevant Human Research Ethics Committees.

\section{Study procedures}

All participants will receive usual care for stroke rehabilitation. Where this involves transfer to inpatient rehabilitation, we will provide transport for participants to return to the laboratory for neurophysiological assessments. In the event that participants are discharged home with residual arm weakness, a physiotherapist will prescribe and supervise a home exercise program based on the GRASP protocol (Graded Repetitive Arm Supplementary Program) [12].

Neurophysiological and functional assessments will be conducted up to eight times over the first year post stroke, as shown in Fig. 1. We anticipate that changes in neuroplasticity will be most evident in the first month post stroke, therefore this will be the period of most intense investigation.

\section{Primary outcome}

We will examine a number of different neurophysiological measures, outlined below, to describe the presence of a critical window. The primary outcome of interest is the change in the motor evoked potential (MEP) amplitude in the hand, after the administration of a plasticity-inducing paradigm to the affected motor cortex. The amplitude of the MEP at any given stimulus intensity reflects the excitability of cortical synapses in the networks generating the MEP. We will use an inhibitory, spaced, continuous theta burst stimulation paradigm (cTBS) to induce short-term plasticity within the motor cortex of the affected hemisphere [13]. We will compare the amplitude of MEPs recorded at baseline, to those recorded at 5, 15, 30 and 45 mins following paired cTBS. The change in MEP amplitude in the affected hemisphere following stroke will be compared to that observed in the dominant hemisphere of age-matched healthy control participants.

\section{Secondary outcomes}

Stimulus-response curves: we will use three stimulus intensities (110, 130 and $150 \%$ resting motor threshold, RMT) to quantify the stimulus-response relationship over time. 
Table 1 Study inclusion and exclusion criteria

\begin{tabular}{l}
\hline Inclusion criteria \\
Experienced a middle cerebral artery stroke with cortical involvement; \\
either a first-ever stroke or at least 1 year after a stroke in non-motor \\
regions \\
Medically stable \\
Confirmed diagnosis of stroke from CT/MRI imaging \\
Have mild/moderate hand weakness (defined in this study as being able \\
to lift and hold a small object, but do not full strength according to \\
Medical Research Council grades) [19] \\
Have recordable motor evoked potentials ( 200 $\mu \mathrm{V}$ ) to transcranial \\
magnetic stimulation (TMS) in affected hand muscles \\
Exclusion criteria \\
Contraindications to TMS [20] \\
For example, history of other neurological disease, including epilepsy, \\
cardiac pacemaker, metal implants in the skull. A complete TMS Safety \\
Screening Questionnaire will be completed in accordance with \\
international guidelines [20] \\
Severe receptive aphasia \\
Pre-morbid dementia \\
Inability to give informed consent
\end{tabular}

Motor threshold: we will record resting and active motor threshold in the first dorsal interosseous muscle of the stroke-affected hand using standard techniques. This provides a measure of excitability in the cortical network activated by TMS (see [14] for review).

Short interval intracortical inhibition (SICI): we will use standard techniques [15] to record the motor cortical intracortical inhibition that occurs when a subthreshold conditioning stimulus (70\% RMT) precedes a supra-threshold test stimulus (120\% RMT), at 2 and $3 \mathrm{~ms}$ inter-stimulus intervals.

We will also perform functional testing to assess rates of recovery of arm function following stroke. These include:

- National Institutes of Health Stroke Scale (NIHSS), a measure of stroke severity, recorded on admission to hospital.

- Functional Independence Measure (FIM), a measure of independence with mobility and activities of daily living.

- The Action Research Arm Test (ARAT), to measure arm activity in the domains pinch, grip, grasp and gross arm movement.

- The Grip-lift task, a sensitive indicator of dextrous hand function following stroke [16].

Finally, we will record the amount of arm movement performed by the affected arm using tri-axial accelerometers (Actiwatches) [17]. These will be worn on both arms for three consecutive days between assessments time points, and will be included in the analysis as a covariate.

\section{Sample size estimate}

Our conservative estimate is for a $5 \%$ increase in the neuroplastic response to cTBS at the optimal timing (i.e. during the critical window) following stroke. Considering the mean response and variance of data from control subjects to the paired cTBS paradigms [13] we would need approximately 43 patients to detect a difference $(\mathrm{p}<0.05)$ at $80 \%$ power. Therefore, to be confident of examining the hypotheses and allowing for a $33 \%$ loss to follow up rate, we will recruit 70 patients over the four years of the grant.

\section{Statistical analyses}

To address our aims we will perform the following analyses:

1. Repeated measures analysis of variance (rmANOVA) to examine changes across time in neuroplasticity (MEP response to CTBS) and inhibition (SICI).

2. ANOVA to compare neuroplasticity and inhibition between patients and controls over time.

3. Generalised Estimating Equations to examine the longitudinal relationship between neuroplasticity, intracortical inhibition, and functional assessment scores.

4. ANOVA comparing neuroplastic responses between patients stratified into tertiles based upon their functional assessment scores (ARAT/FIM) at the end of the trial period (poor recovery/moderate recovery/good recovery).

Where appropriate, lesion size/site (using assessment of corticospinal tract structure from automated segmentation of structural MRI) and upper limb activity scores will be used as covariates in the above analyses.

\section{Study organisation and funding}

This study has been funded by the National Health and Medical Research Council (NHMRC) of Australia, Project Grant ID 1058639, from 2014-2017 and partially funded by a Medical Research Council (UK) Grant MR/ K01384X/1 to JCR.

\section{Discussion}

Little is known about the time course of neuroplastic potential in the damaged hemisphere following stroke, nor whether this impacts upon the effectiveness of rehabilitative strategies and true restitution of function. We will carefully examine changes in the corticospinal pathway, with MEP amplitude and stimulus response curves, in individuals from approximately one week to one year post stroke. We will also describe changes in intracortical inhibition, thought to influence functional recovery in the brain post stroke [18]. Most importantly, we will perform serial investigations of the response of the affected motor cortex to 
paired cTBS, designed to induce short-term plasticity in the motor cortex. Comparing this response to aged-matched controls, as well as longitudinal assessment of functional recovery, will provide definitive evidence, or lack thereof, for a critical window of neuroplasticity following stroke.

\section{Summary and conclusions}

This study will further our understanding of the changes that occur in the damaged brain that support functional recovery following stroke. Optimising rehabilitation to a proposed critical window of neuroplasticity could help to improve both speed and the amount of recovery following stroke and could lead to significantly greater improvements in functional outcomes.

\begin{abstract}
Abbreviations
ARAT: Action Research Arm Test; FIM: Functional Independence Measure; GABA: Gamma-aminobutyric acid; GRASP: Graded Repetitive Arm Supplementary Program; MEP: Motor evoked potential; MRI: Magnetic resonance imaging; NIHSS: National Institutes of Health Stroke Scale; rmANOVA: Repeated measures analysis of variance; RMT: Resting motor threshold; SICl: Short-interval intracortical inhibition; TBS: Theta burst stimulation; TMS: Transcranial Magnetic Stimulation.
\end{abstract}

\section{Competing interests}

The authors declare that they have no competing interests.

\section{Authors' contributions}

JCR and MCR conceived the study, SK, NW and MMCD and SK contributed to the study design and $\mathrm{BH}$ and MCR are primarily responsible for data collection. All authors, with the exception of $\mathrm{BH}$, contributed to the funding application. All authors read and approved the final manuscript.

\section{Acknowledgements}

This study has received funding by the National Health and Medical Research Council (NHMRC) of Australia, Project Grant ID 1058639, from 2014-2017 and partially funded by a Medical Research Council (UK) Grant MR/K01384X/1 to JCR. Neither funding body has any role in the design, collection, analysis or interpretation of data. MCR is supported by the University of Adelaide as a University Senior Research Fellow. We thank Dr Ann-Maree Vallence for assistance with data collection in 2014.

\section{Author details}

${ }^{1}$ Alliance for Research in Exercise, Nutrition and Activity and International Centre for Allied Health Evidence, Sansom Institute for Health Research, School of Health Sciences, University of South Australia, GPO Box 2471, Adelaide, SA 5001, Australia. ${ }^{2}$ Stroke Research Programme, School of Medicine, South Australian Health and Medical Research Institute, University of Adelaide, Adelaide, Australia. ${ }^{3}$ Sobell Department of Motor Neuroscience, University College London Institute of Neurology, Queen Square, London, England. ${ }^{4}$ UCLP Centre For Neurorehabilitation, Queen Square, London, England. ${ }^{5}$ The National Hospital for Neurology and Neurosurgery, Queen Square, London, England. ${ }^{6}$ Neuromotor Plasticity and Development Research Group, Robinson Research Institute, School of Paediatrics and Reproductive Health, University of Adelaide, Adelaide, Australia.

Received: 29 May 2015 Accepted: 18 June 2015

Published online: 11 July 2015

\section{References}

1. Dancause N, Barbay S, Frost SB, Plautz EJ, Chen D, Zoubina EV, et al. Extensive cortical rewiring after brain injury. J Neurosci. 2005;25(44): 10167-79.

2. Kwakkel G, Kollen B, Lindeman E. Understanding the pattern of functional recovery after stroke: facts and theories. Restor Neurol Neurosci. 2004; 22(3-5):281-99.
3. Fawcett J. Molecular control of brain plasticity and repair. Prog Brain Res. 2009:175:501-9.

4. Weiller C, Juptner M, Fellows S, Rijntjes M, Leonhardt G, Kiebel S, et al. Brain representation of active and passive movements. Neuroimage. 1996;4(2):105-10.

5. Nelles G, Spiekermann G, Jueptner M, Leonhardt G, Muller S, Gerhard H, et al. Reorganization of sensory and motor systems in hemiplegic stroke patients. A positron emission tomography study. Stroke. 1999;30(8):1510-6.

6. Ward NS, Brown MM, Thompson AJ, Frackowiak RS. Neural correlates of motor recovery after stroke: a longitudinal fMRI study. Brain. 2003;126(Pt 11):2476-96.

7. Neumann-Haefelin T, Hagemann G, Witte OW. Cellular correlates of neuronal hyperexcitability in the vicinity of photochemically induced cortical infarcts in rats in vitro. Neurosci Lett. 1995;193(2):101-4.

8. Hagemann G, Redecker C, Neumann-Haefelin T, Freund HJ, Witte OW Increased long-term potentiation in the surround of experimentally induced focal cortical infarction. Ann Neurol. 1998:44(2):255-8.

9. Cicinelli P, Pasqualetti P, Zaccagnini M, Traversa R, Oliveri M, Rossini PM. Interhemispheric asymmetries of motor cortex excitability in the postacute stroke stage: a paired-pulse transcranial magnetic stimulation study. Stroke 2003:34(11):2653-8.

10. Manganotti P, Patuzzo S, Cortese F, Palermo A, Smania N, Fiaschi A. Motor disinhibition in affected and unaffected hemisphere in the early period of recovery after stroke. Clin Neurophysiol. 2002;113(6):936-43.

11. Salter K, Jutai J, Hartley M, Foley N, Bhogal S, Bayona N, et al. Impact of early vs delayed admission to rehabilitation on functional outcomes in persons with stroke. J Rehabil Med. 2006;38(2):113-7.

12. Harris JE, Eng JJ, Miller WC, Dawson AS. A self-administered Graded Repetitive Arm Supplementary Program (GRASP) improves arm function during inpatient stroke rehabilitation: a multi-site randomized controlled trial. Stroke. 2009;40(6):2123-8.

13. Goldsworthy MR, Pitcher JB, Ridding MC. The application of spaced theta burst protocols induces long-lasting neuroplastic changes in the human motor cortex. Eur J Neurosci. 2012;35(1):125-34.

14. Ziemann U. TMS and drugs. Clin Neurophysiol. 2004;115(8):1717-29.

15. Ridding MC, Taylor $\mathrm{J}$, Rothwell JC. The effect of voluntary contraction on cortico-cortical inhibition in human motor cortex. J Physiol. 1995;487(Pt 2):541-8.

16. McDonnell MN, Hillier SL, Ridding MC, Miles TS. Impairments in precision grip correlate with functional measures in adult hemiplegia. Clin Neurophysiol. 2006;117(7):1474-80.

17. van der Pas SC, Verbunt JA, Breukelaar DE, van Woerden R, Seelen HA Assessment of arm activity using triaxial accelerometry in patients with a stroke. Arch Phys Med Rehabil. 2011;92(9):1437-42.

18. Swayne OB, Rothwell JC, Ward NS, Greenwood RJ. Stages of motor output reorganization after hemispheric stroke suggested by longitudinal studies of cortical physiology. Cereb Cortex. 2008;18(8):1909-22.

19. Medical Research Council. Aids to the examination of the peripheral nervous system. London: Bailliere Tindall; 1990.

20. Rossi S, Hallett M, Rossini PM, Pascual-Leone A. Safety, ethical considerations, and application guidelines for the use of transcranial magnetic stimulation in clinical practice and research. Clin Neurophysiol. 2009;120(12):2008-39.

\section{Submit your next manuscript to BioMed Central and take full advantage of:}

- Convenient online submission

- Thorough peer review

- No space constraints or color figure charges

- Immediate publication on acceptance

- Inclusion in PubMed, CAS, Scopus and Google Scholar

- Research which is freely available for redistribution 